Anaesthesist 2019 $68: 770-776$ https://doi.org/10.1007/s00101-019-00640-5 Online publiziert: 22. August 2019 (c) Springer Medizin Verlag GmbH, ein Teil von Springer Nature 2019

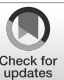

M. Dietrich ${ }^{1}$ C. J. Reuß ${ }^{1}$ C. Beynon ${ }^{2}$ A. Hecker ${ }^{3}$. C. Jungk ${ }^{2} \cdot$ D. Michalski ${ }^{4}$.

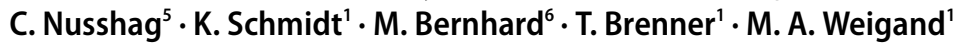

${ }^{1}$ Klinik für Anästhesiologie, Universitätsklinikum Heidelberg, Heidelberg, Deutschland ${ }^{2}$ Neurochirurgische Klinik, Universitätsklinikum Heidelberg, Heidelberg, Deutschland ${ }^{3}$ Klinik für Allgemein- Viszeral-, Thorax-, Transplantations- und Kinderchirurgie, Universitätsklinikum Gießen und Marburg, Standort Gießen, Gießen, Deutschland

${ }^{4}$ Neurologische Intensivstation und Stroke Unit, Klinik und Poliklinik für Neurologie, Universitätsklinikum Leipzig AöR, Leipzig, Deutschland

${ }^{5}$ Klinik für Endokrinologie, Stoffwechsel und klinische Chemie/Sektion Nephrologie, Universitätsklinikum Heidelberg, Heidelberg, Deutschland

${ }^{6}$ Zentrale Notaufnahme, Universitätsklinikum Düsseldorf, Düsseldorf, Deutschland

\title{
Beatmung und Sauerstofftherapie
}

\section{Intensivmedizinische Studien aus 2018/2019}

\section{Infobox}

Dieser Beitrag ist Teil einer Serie zu den wichtigsten Intensivmedizinische Studien aus 2018/2019. Weitere Teile der Serie sind:

- Editorial: Coburn M (2019) Intensivmedizinische Studien aus 2018/2019. Anaesthesist. https://doi.org/10.1007/ s00101-019-00638-z

- Flüssigkeitstherapie: Reuß CJ, Dietrich M, Beynon C et al (2019) Flüssigkeitstherapie. Intensivmedizinische Studien aus 2018/2019. Anaesthesist. https://doi.org/ 10.1007/s00101-019-00639-y

- Fokus Nephrologie: Nusshag C, Beynon C, Dietrich M et al (2019) Fokus Nephrologie. Intensivmedizinische Studien aus 2018/2019. Anaesthesist. https://doi.org/ 10.1007/s00101-019-00641-4

- Additive Therapien: Dietrich M, Reuß CJ, Beynon C et al (2019) Additive Therapien. Intensivmedizinische Studien aus 2018/2019. Anaesthesist. https://doi.org/ 10.1007/s00101-019-00642-3

- Neurologische Intensivmedizin: Michalski D, Jungk C, Brenner T et al (2019) Neurologische Intensivmedizin. Intensivmedizinische Studien aus 2018/2019. Anaesthesist. https://doi.org/10.1007/ s00101-019-00643-2

- Fokus Neurochirurgie: Beynon C, Bernhard M, Brenner T et al (2019) Fokus Neurochirurgie. Intensivmedizinische Studien aus 2018/2019. Anaesthesist. https://doi.org/10.1007/s00101-01900644-1
Da viele Studien unter Akronymen ,gehandelt" werden, gibt - Tab. 1 einen Überblick über die im vergangenen Jahr erschienenen Studien unter gleichzeitiger Nennung der jeweiligen Akronyme (sofern vorhanden).

Das „acute respiratory distress syndrome" (ARDS) beschreibt ein akutes Lungenversagen, dem verschiedene Ursachen zugrunde liegen können. Die Diagnosestellung und Einteilung in 3 Schweregrade erfolgt nach den Berlin-Kriterien aus dem Jahr 2012 unter Berücksichtigung des Horovitz-Quotienten (arterieller Sauerstoffpartialdruck $\left(\mathrm{p}_{\mathrm{a}} \mathrm{O}_{2}\right)$, dividiert durch die inspiratorische Sauerstofffraktion $\left.\left(\mathrm{F}_{\mathrm{I}} \mathrm{O}_{2}\right)\right)$ (• Tab. 2; [2]):

Die Sterblichkeit des ARDS ist hoch und variiert je nach Erkrankungsschwere von 27 bis über $60 \%$ [3-6]. Ein ARDS kann als Reaktion auf eine Systemerkrankung auftreten oder Folge einer direkten Lungenschädigung sein. Durch die direkte oder indirekte Schädigung kommt es durch die Freisetzung proinflammatorischer Zytokine zu einer überschießenden Immunreaktion mit Invasion neutrophiler Granulozyten. Diese setzen zytotoxische Substanzen und reaktive Sauerstoffspezies frei; ein Prozess, der zu einer erhöhten vaskulären Permeabilität mit konsekutivem, alveolärem Ödem und über einen Surfactant-Mangel zur Atelektasenbildung führt [7].
Zur Therapie der Hypoxämie bis zur Erholung der Lungenfunktion ist in vielen Fällen eine invasive Beatmung notwendig. Um eine weitere ventilatorassoziierte Lungenschädigung zu vermeiden, ist die konsequente Umsetzung lungenprotektiver Beatmungsregime essenziell $[1,8]$ :

- Tidalvolumen: maximal $6 \mathrm{ml} / \mathrm{kg}$ ideales Körpergewicht,

- inspiratorische Druckdifferenz (,driving pressure“) $\leq 15 \mathrm{~cm} \mathrm{H}_{2} \mathrm{O}$,

- maximaler inspiratorischer Spitzendruck möglichst bei $<30$ mbar,

- Einstellung eines adäquaten positiven endexspiratorischen Beatmungsdruckes (,positive end-expiratory pressure", PEEP),

- ggf. Bauchlage,

- ggf. Relaxierung.

\section{Originalpublikation}

Combes A, Hajage D, Capellier G et al (2018) Extracorporeal membrane oxygenation for Severe Acute Respiratory Distress Syndrome. N Engl J Med 378:1965-1975

Die Anwendung der extrakorporalen Membranoxygenierung (ECMO) als „Rescue“-Lungenersatzverfahren gewann über die letzten Jahre zunehmend an Bedeutung. Die Anwendungszah- 
Tab. 1 In den Jahren 2018/2019 erschienene intensivmedizinische Studien und die Bedeutung der Akronyme

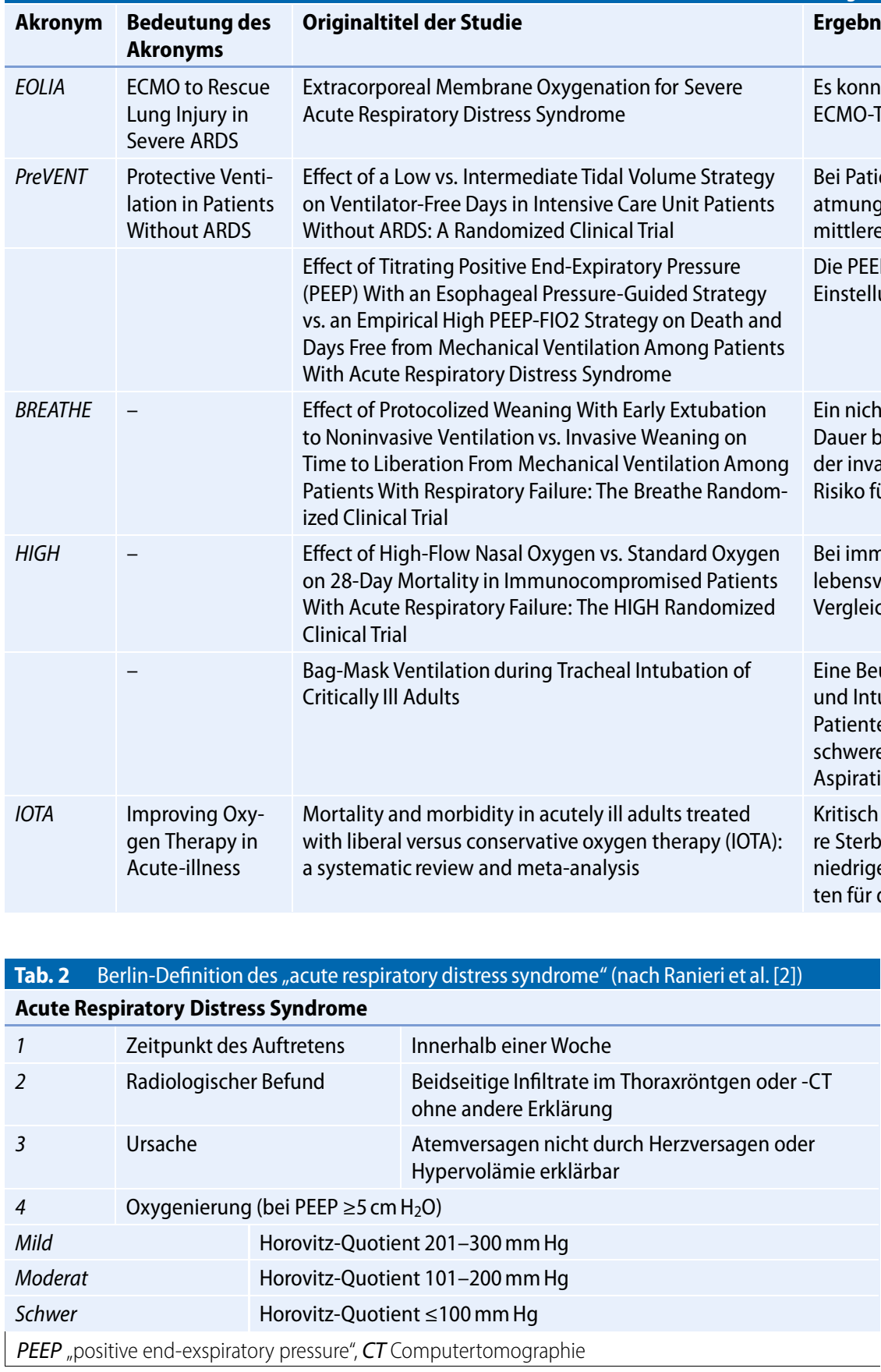

len und die Anzahl der Zentren, in denen eine ECMO eingesetzt werden kann, nahmen trotz der hohen Therapiekosten über die letzten Jahre hinweg stetig zu $[9,10]$. Die Frage, ob über den Einsatz der ECMO eine Verbesserung der Prognose erreicht werden kann, wird derzeit kontrovers diskutiert. Eine randomisierte, kontrollierte Studie von Peek et al. [11] aus dem Jahr 2009 zeigte vielversprechende Ergebnis- se für eine Prognoseverbesserung durch eine ECMO-Therapie, jedoch bei eingeschränkter Aussagekraft aufgrund des methodischen Vorgehens der Studie. Die EOLIA-Studie [12] befasste sich ebenso mit dieser Fragestellung. In die randomisierte, kontrollierte Multizenterstudie wurden 249 Patienten mit schwerem ARDS eingeschlossen. Die Diagnosestellung des ARDS erfolgte anhand der American-European-Consensus-Confe-
rence-Definition von 1994, da die Studie vor Veröffentlichung der Berlin-Definition aus dem Jahr 2011 geplant wurde [13]. Zusätzlich mussten die Patienten bei Einschluss in die Studie für weniger als 7 Tage mechanisch beatmet worden sein und mindestens eines der folgenden 3 Kriterien erfüllen:

- Horovitz-Quotient $<50 \mathrm{~mm}$ Hg über $3 \mathrm{~h}$,

- Horovitz-Quotient $<80 \mathrm{~mm}$ Hg über $6 \mathrm{~h}$,

- pH-Wert im arteriellen Blut $<7,25$ mit einem $\mathrm{pCO}_{2}>60 \mathrm{~mm} \mathrm{Hg}$ über $6 \mathrm{~h}$.

Es erfolgte die Randomisierung in 2 Gruppen: Bei der ECMO-Gruppe wurde umgehend die ECMO-Therapie begonnen. Die Kontrollgruppe wurde weiterhin konventionell mittels lungenprotektiver Beatmung therapiert, jedoch war ein Wechsel auf eine „Rescue“ECMO-Therapie möglich, wenn die Sauerstoffsättigung über $6 \mathrm{~h}$ unter $80 \%$ lag, kein irreversibles Organversagen vorlag und der behandelnde Arzt der 
Meinung war, dass das Behandlungsergebnis des Patienten durch eine ECMOTherapie verbessert werden könnte.

Der primäre Endpunkt der Studie war die 60-Tage-Sterblichkeit, während das Therapieversagen (definiert als Beginn einer ECMO-Therapie in der konventionellen Gruppe oder Versterben der Patienten), die Sterblichkeit zu verschiedenen Zeitpunkten und diverse ECMOassoziierte Komplikationen sekundäre Endpunkte darstellten.

Die Studie wurde nach der vierten Interimsanalyse von 249 Patienten abgebrochen, da sich bezüglich des primären Endpunkts kein signifikanter Unterschied zeigte. Bei Randomisierung waren die beiden Gruppen in Bezug auf die Baseline-Daten vergleichbar.

Die Sterblichkeit nach 60 Tagen lag bei $35 \%$ in der ECMO-Gruppe und bei $46 \%$ in der Kontrollgruppe $(p=0,09)$. Bei 35 Patienten (28\%) der Kontrollgruppe wurde eine „Rescue“-ECMOTherapie durchgeführt, sodass sich die Gruppen signifikant bezüglich des sekundären Endpunkts Therapieversagen unterschieden: ECMO-Gruppe $35 \%$ vs. Kontrollgruppe $58 \%(p<0,001)$. Zwanzig von 35 Patienten (57\%) der Kontrollgruppe, bei denen eine "Rescue“ECMO-Therapie durchgeführt werden musste, verstarben im Rahmen des Behandlungsverlaufs.

Durch die EOLIA-Studie konnte kein signifikanter Überlebensvorteil für die ECMO bei Patienten mit schwerem ARDS gezeigt werden. Um einen signifikanten Vorteil zu zeigen, wäre mit der angenommenen Studien-Power eine absolute Reduktion der Sterblichkeit von $20 \%$ durch die ECMO notwendig gewesen. Jedoch ergeben sich in einer nachträglich durchgeführten BayesAnalyse (ein statistisches Vorgehen, mit dem man die Wahrscheinlichkeit für eine Risikoreduktion errechnen kann) deutliche Hinweise für einen Überlebensvorteil der ECMO-Therapie [14]. Außerdem könnten die Ergebnisse zum Nachteil der ECMO-Gruppe durch die Möglichkeit der „Rescue“-ECMO-Therapie in der Kontrollgruppe beeinflusst worden sein.

Die Frage, ob für die ECMO-Therapie ein signifikanter Überlebensvorteil in
Studien gezeigt werden kann, bleibt also auch nach der EOLIA-Studie unbeantwortet [12]. Entsprechend dem Studienprotokoll der EOLIA-Studie liegt es jedoch in vielen Fällen eines schweren ARDS nahe, eine ECMO als „Rescue“Therapie anzuwenden. Entscheidend ist es, individuell für jeden Patienten den richtigen Zeitpunkt für den Beginn einer ECMO-Therapie zu finden.

\section{Originalpublikation}

Simonis FD, Serpa Neto A, Binnekade JM et al (2018) Effect of a low vs. intermediate tidal volume strategy on ventilator-free days in intensive care unit patients without ARDS: a randomized clinical trial. JAMA 320:1872-1880

Bei einem ARDS führt eine lungenprotektive Beatmungsstrategie mit niedrigem Tidalvolumen (6-7 ml/kgKG), verglichen mit einer Beatmung mit höheren Tidalvolumina (10-15 ml/kgKG), zu einem Überlebensvorteil [15]. Es ist bisher allerdings unklar, ob Patienten, die nicht an einem ARDS leiden, ebenfalls von einer Beatmung mit niedrigen Tidalvolumina profitieren. Eine multizentrische, randomisierte, kontrollierte Studie aus den Niederlanden beschäftigte sich mit der Fragestellung, ob eine Beatmung mit niedrigen Tidalvolumina (4-6 ml/kgKG) einer Beatmung mit höheren Tidalvolumina $(10 \mathrm{ml} / \mathrm{kgKG})$ bezüglich der beatmungsfreien Tage innerhalb von $28 \mathrm{Ta}$ gen nach Aufnahme auf Intensivstation überlegen ist (PReVENT-Studie) [16]. Es wurden chirurgische und internistische Patienten, bei denen eine Beatmungsdauer über 24 h erwartet wurde und nach der Berlin-Definition kein ARDS vorlag, eingeschlossen. Sekundäre Endpunkte waren die Sterblichkeit, die Dauer des Krankenhaus- und Intensivaufenthalts und die Entwicklung eines ARDS, einer Pneumonie, einer schweren Atelektase sowie eines Pneumothorax. Die Gruppe mit niedrigen Tidalvolumina umfasste 475 Patienten und wurde mit einem Tidalvolumen von $6 \mathrm{ml} / \mathrm{kgKG}$ unter Nutzung einer volumenkontrollierten Beatmung oder eines druckunterstützten Spontanbeatmungsmodus beatmet. Sofern möglich, wurde anschließend das Tidalvolumen auf $4 \mathrm{ml} /$ kgKG reduziert. Die minimale Druckun- terstützung lag bei $5 \mathrm{~cm} \mathrm{H}_{2} \mathrm{O}$. Eine Spontanatmung mit höheren Tidalvolumina wurde toleriert. In die Gruppe mit höheren Tidalvolumina wurden 480 Patienten eingeschlossen, für die ein maximaler Plateaudruck von $25 \mathrm{~cm} \mathrm{H}_{2} \mathrm{O}$ festgelegt wurde. Falls dieser überschritten werden musste, reduzierte man das Tidalvolumen, um den Grenzwert nicht $\mathrm{zu}$ überschreiten. Um das Studienprotokoll einzuhalten, wurden keine zusätzliche Analgosedierung oder Muskelrelaxation durchgeführt.

Insgesamt wurden 961 Patienten auf 6 Intensivstationen randomisiert (477 Patienten mit niedrigen Tidalvolumina vs. 484 Patienten mit höheren Tidalvolumina). Bezüglich des primären Endpunkts zeigte sich im Median kein Unterschied zwischen den beiden Gruppen (21 vs. 21 beatmungsfreie Tage bis Tag 28). Auch bezüglich der sekundären Endpunkte konnte kein signifikanter Unterschied gezeigt werden. Patienten der Gruppe mit niedrigem Tidalvolumen hatten einen signifikant höheren $\mathrm{CO}_{2}$-Partialdruck und einen niedrigeren Blut-pHWert.

Die PReVENT-Studie [16] konnte keinen Vorteil für eine Beatmungsstrategie mit niedrigem Tidalvolumen für Patienten ohne ARDS zeigen. Jedoch wurden auch in der Kontrollgruppe hohe Plateaudrücke $\left(>25 \mathrm{~cm} \mathrm{H}_{2} \mathrm{O}\right)$ und damit exzessive Barotraumata vermieden. Daraus ergibt sich, dass bei Patienten ohne ARDS eine Hyperkapnie und eine daraus folgende respiratorische Acidose nicht zugunsten kleiner Tidalvolumina toleriert werden müssen, zumindest, solange hohe Spitzendrücke vermieden werden. Generell können Patienten ohne ARDS nach den Ergebnissen der PReVENT-Studie mit moderaten Tidalvolumina $(6-8 \mathrm{ml} /$ $\mathrm{kgKG)}$ beatmet werden.

\section{Originalpublikation}

Beitler JR, Sarge T, Banner-Goodspeed VM et al (2019) Effect of titrating Positive End-Expiratory Pressure (PEEP) with an esophageal pressure-guided strategy vs. an empirical high PEEP-FiO2 strategy on death and days free from mechanical ventilation among patients with Acute Respiratory Distress Syndrome. JAMA 321:846-857 
Eine optimale Einstellung des positiven endexspiratorischen Drucks (PEEP) soll ein Kollabieren der Alveolen in der Exspirationsphase verhindern und dadurch die beatmungsassoziierte Schädigung der Lunge reduzieren. Wird ein zu hoher PEEP gewählt, kann dies über hohe Beatmungsdrücke ebenfalls zu einer Schädigung des Lungengewebes führen.

Eine prospektive, randomisierte, kontrollierte Studie, in die Patienten mit mildem bis schwerem ARDS von 14 nordamerikanischen Intensivstation eingeschlossen wurden, untersuchte, ob eine PEEP-Titration anhand des Ösophagusdrucks einer Einstellung des PEEP nach einer empirischen Tabelle überlegen ist [17]. Eine Studie von Talmor et al. [18] konnte 2008 eine verbesserte Oxygenierung und Compliance für eine Einstellung anhand des Ösophagusdrucks zeigen.

Primär wurde ein kombinierter Endpunkt aus Überleben und beatmungsfreien Tagen bis zum 28. Tag nach Einschluss in die Studie evaluiert. Der Ösophagusdruck entspricht näherungsweise dem intrapleuralen Druck. In der Interventionsgruppe wurde der PEEP so gewählt, dass der transpulmonale Druck (Atemwegsdruck minus Pleuradruck) zwischen $0-6 \mathrm{~cm} \mathrm{H}_{2} \mathrm{O}$ lag, nie jedoch niedriger oder wesentlich höher. Das Atemzugvolumen wurde zwischen $4-8 \mathrm{ml} / \mathrm{kgKG}$ gewählt und bei endinspiratorischem transpulmonalem Druck $>20 \mathrm{cmH}_{2} \mathrm{O}$ reduziert bzw. bei Dyspnoe oder Acidose erhöht. In der Kontrollgruppe wurde die niedrigste mögliche Kombination nach einer empirischen $\mathrm{F}_{\mathrm{I}} \mathrm{O}_{2}$-PEEP-Tabelle gewählt, um eine ausreichende Oxygenierung $\mathrm{zu}$ gewährleisten. Eingeschlossen wurden insgesamt 200 Patienten (Interventionsgruppe: $n=102$, Kontrollgruppe: $n=98$ ).

Hinsichtlich des primären Endpunkts zeigte sich kein Unterschied zwischen den Studiengruppen $(p=0,92)$. Nach 28 Tagen waren 33 Patienten $(32,4 \%)$ der ösophagusdruckgesteuerten Gruppe und 30 Patienten $(30,6 \%)$ der Kontrollgruppe verstorben $(p=0,88)$. Bezüglich beatmungsfreier Tage und sonstiger sekundärer Endpunkte gab es ebenfalls keine signifikanten Unterschiede. Die beiden Studiengruppen wurden trotz verschiedener Vorgehensweisen bei der Einstellung des PEEP jedoch sehrähnlich beatmet. Bezüglich der Beatmungsparameter, des endexspiratorischen Ösophagusdrucks, des PEEP, des transpulmonalen endinspiratorischen Plateaudrucks, der „driving pressure“ sowie des Horovitz-Quotienten zeigten sich im Mittel innerhalb der ersten 7 Studientage keine signifikanten Differenzen. Basierend auf den Ergebnissen dieser Studie ist eine PEEP-Titration anhand empirischer Tabellen, wie in diesem Fall diejenige der Kontrollgruppe der OSCILLATEStudie [19], der Einstellung anhand des Ösophagusdrucks gleichwertig.

\section{Originalpublikation}

Perkins GD, Mistry D, Gates S et al (2018) Effect of protocolized weaning with early extubation to noninvasive ventilation vs. invasive weaning on time to liberation from mechanical ventilation among patients with respiratory failure: the breathe randomized clinical trial. JAMA 320:1881-1888

Die Entwöhnung von der invasiven Beatmung sollte möglichst frühzeitig nach standardisierten Protokollen durchgeführt werden [20]. Eine randomisierte, kontrollierte Studie aus Großbritannien [21] untersuchte, ob bei Patienten mit komplizierter Beatmungsentwöhnung die Extubation mit anschließender, nichtinvasiver Beatmung die Dauer bis zur Entwöhnung von jeglicher Beatmung, im Vergleich zu konventionellen Weaning-Protokollen, verkürzen kann. Dafür wurden 364 Patienten auf 41 Intensivstationen eingeschlossen, die mindestens $48 \mathrm{~h}$ über einen endotrachealen Tubus beatmet wurden und bei denen ein Versuch der Spontanatmung fehlgeschlagen war. Das konventionelle, invasive Weaning-Protokoll sah eine druckunterstützte Spontanatmung über den Tubus vor. Die Druckunterstützung wurde im Intervall von $2 \mathrm{~h} \mathrm{um} 2 \mathrm{~cm} \mathrm{H}_{2} \mathrm{O}$ reduziert, wenn der Patient keine Anzeichen für Dyspnoe oder respiratorische Erschöpfung zeigte. Ansonsten wurde die Druckunterstützung um $2 \mathrm{~cm} \mathrm{H}_{2} \mathrm{O}$ erhöht. War der täglich durchgeführte Spontanatmungsversuch erfolgreich, erfolgte die Extubation.
Bei dem nichtinvasiven Vorgehen erfolgte die Extubation mit Wechsel auf eine nichtinvasive Beatmung mittels Beatmungsmaske bereits, sobald der behandelnde Arzt ein Weaning für möglich hielt. Es wurden am Respirator zunächst die gleichen Einstellungen, wie bei der invasiven Beatmung beibehalten, und es wurde ebenfalls alle $2 \mathrm{~h}$ reevaluiert. Bezüglich der Dauer bis zur Beatmungsfreiheit zeigte sich kein signifikanter Unterschied zwischen den Gruppen. Jedoch waren beim nichtinvasiven Protokoll die Dauer der invasiven Beatmung (1 vs. 4 Tage) und die Anzahl der Beatmungstage (3 vs. 4 Tage) signifikant reduziert. Zudem erhielten die Patienten der nichtinvasiven Weaning-Gruppe weniger antibiotische Therapie aufgrund von respiratorischen Infektionen $(60,4 \%$ vs. $70,3 \%)$. Dabei zeigte sich kein signifikanter Unterschied bezüglich Sterblichkeit, Reintubations- oder Tracheostomierate und dem Auftreten unerwünschter Ereignisse [21].

Ein nichtinvasives Weaning-Protokoll verkürzt also nicht die Zeit bis zur Entwöhnung von der Beatmung (invasiv und nichtinvasiv). Die Dauer der invasiven Beatmung kann dadurch aber ohne zusätzliches Risiko für den Patienten verkürzt werden.

\section{Originalpublikation}

Azoulay E, Lemiale V, Mokart D et al (2018) Effect of high-flow nasal oxygen vs. standard oxygen on 28-day mortality in immunocompromised patients with acute respiratory failure: the HIGH randomized clinical trial. JAMA 320:2099-2107

Eine invasive Beatmung sollte besonders bei immunsupprimierten Patienten vermieden werden. Eine Möglichkeit zur Behandlung einer akuten Oxygenierungsstörung ist die „High-flow“-Sauerstofftherapie [22]. Die randomisierte, multizentrische HIGH-Studie [23] untersuchte auf 32 Intensivstationen in Frankreich den Einfluss einer High-flow-Sauerstofftherapie auf die 28-Tage-Sterblichkeit bei immunsupprimierten Patienten mit hypoxämischem, respiratorischem Versagen im Vergleich zu einer Standardsauerstofftherapie. Für den Einschluss in die 


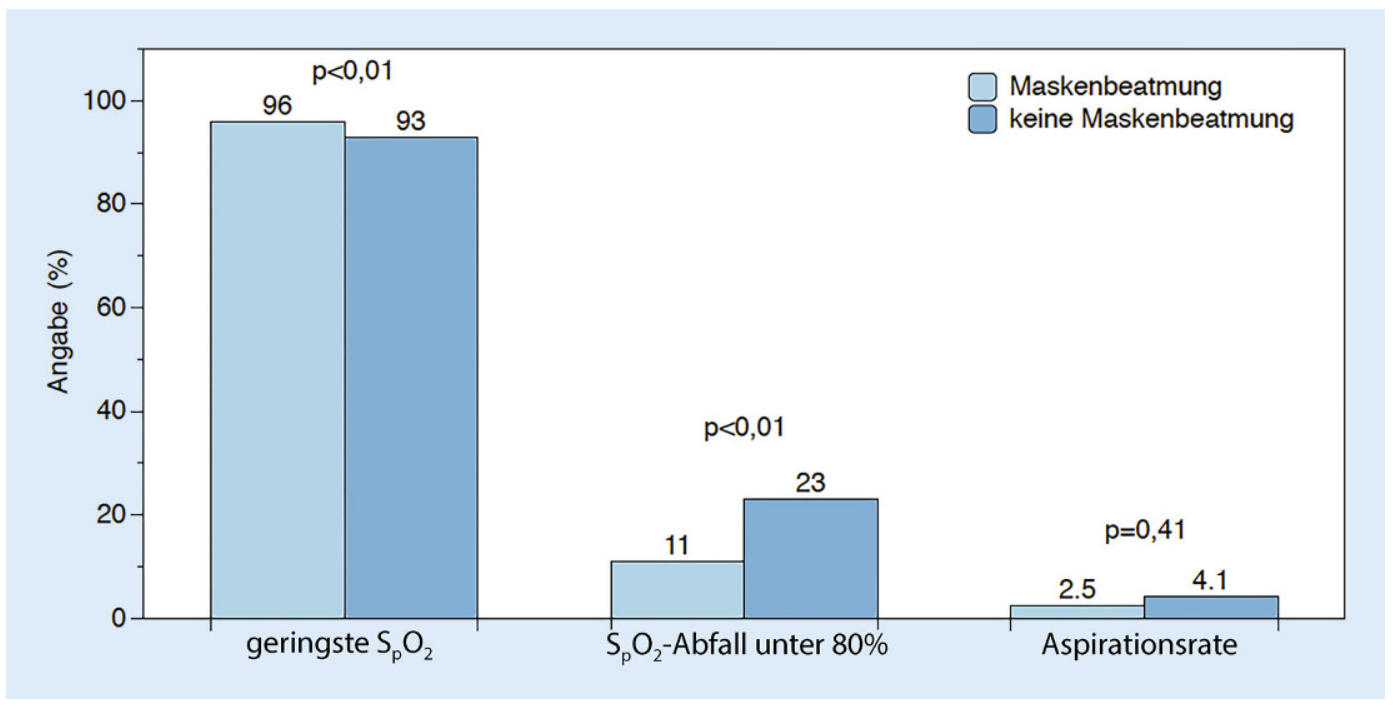

Abb. $1<$ Sauerstoffsättigung $\left(\mathrm{S}_{\mathrm{p}} \mathrm{O}_{2}\right)$ und Aspirationsrate der Gruppen mit und ohne Maskenbeatmung
Studie mussten folgende Kriterien erfüllt sein:

1. Immunsuppression, definiert als: Langzeit- (>3 Monate) oder Hochdosissteroideinnahme $(>0,5 \mathrm{mg} / \mathrm{kgKG}$ und Tag), sonstige Immunsuppressivaeinnahme, Z.n. Organtransplantation, Tumorerkrankung mit Chemotherapie in den vergangenen 5 Jahren, maligne hämatologische Erkrankungen oder primäre Immundefizienz;

2. hypoxämisches respiratorisches Versagen definiert als: Sauerstoffpartialdruck $<60 \mathrm{~mm} \mathrm{Hg}$ oder Sauerstoffsättigung $<90 \%$ bei Raumluft, Tachypnoe $>30 /$ min oder klinische Zeichen der Atemnot.

Die High-flow-Sauerstofftherapie wurde mit einem Flow von 501/min und einer $\mathrm{F}_{\mathrm{I}} \mathrm{O}_{2}$ von $100 \%$ begonnen; anschließend wurde diese mit dem Ziel einer Sauerstoffsättigung von $95 \%$ angepasst. Auch in der Kontrollgruppe mit Standardsauerstofftherapie wurde die Flussrate (maximal 151/min) entsprechend gewählt, um eine Zielsättigung von $95 \% \mathrm{zu}$ erreichen.

Insgesamt wurden 778 Patienten randomisiert. Sowohl bezüglich der 28Tage-Sterblichkeit (High flow 35,6\% vs. Standard 36,1\%) als auch der Sterblichkeit auf der Intensivstation und im Krankenhaus zeigten sich keine Unterschiede zwischen den beiden Gruppen. Auch die Anzahl der Patienten, die invasivbeatmet werden mussten, unterschied sich nicht signifikant.
Die HIGH-Studie konnte damit bei immunsupprimierten Patienten keinen Überlebensvorteil einer High-flow-Sauerstofftherapie im Vergleich zu einer Standardsauerstofftherapie nachweisen [23].

\section{Originalpublikation}

Casey JD, Janz DR, Russell DW et al (2019) Bagmask ventilation during tracheal intubation of critically III adults. N Engl J Med 380:811-821

Stellt eine endotracheale Intubation bei kritisch kranken Patienten auf der Intensivstation ein besonderes Risiko dar? Durch eine vorgeschädigte Lunge, Sepsis und zeitkritische Intubationssituationen kann es einerseits schneller $\mathrm{zu}$ einer Hypoxämie mit Abfall der Sauerstoffsättigung kommen, andererseits besteht ein erhöhtes Aspirationsrisiko durch gastrointestinale Blutungen, fehlende Nüchternheit und Passagestörungen. $\mathrm{Ob}$ eine Beutel-Maske-Beatmung zwischen Narkoseeinleitung und Intubation bei kritisch kranken Patienten durchgeführt werden soll, wird daher kontrovers diskutiert.

Eine amerikanische, randomisierte, kontrollierte Studie zu dieser Fragstellung wurde im Februar 2019 publiziert [24]. Bei der Interventionsgruppe wurde eine Beutel-Maske-Beatmung zwischen Narkoseeinleitung und Intubation durchgeführt, bei der Kontrollgruppe erfolgte die klassische „rapid sequence induction“ ohne Zwischenbeatmung.
Primärer Endpunkt war die tiefste, gemessene Sauerstoffsättigung von Beginn der Narkoseeinleitung bis $2 \mathrm{~min}$ nach endotrachealer Intubation. Sekundärer Endpunkt war der Anteil an Patienten, die mit der Sauerstoffsättigung auf Werte unter $80 \%$ abfielen. Ausschlusskriterien waren Schwangerschaft, mechanischer Ileus oder wenn der behandelnde Arzt die Entscheidung für oder gegen eine Maskenbeatmung getroffen hatte und somit keine Randomisierung stattfinden konnte.

In die Studie wurden insgesamt 401 Patienten eingeschlossen. Eine BeutelMaske-Beatmung wurde bei 199 Patienten durchgeführt. Der Median der tiefsten Sauerstoffsättigung lag bei $96 \%$. $10,9 \%$ der Patienten fielen mit der Sauerstoffsättigung auf Werte unter $80 \%$ ab. In die Gruppe ohne Zwischenbeatmung wurden 202 Patienten eingeschlossen. Der Median der tiefsten Sauerstoffsättigung lag hier bei $93 \%$. 22,8\% dieser Patienten fielen mit der Sauerstoffsättigung auf Werte unter $80 \%$ ab. Es konnte damit ein signifikanter Vorteil für eine Beutel-Maske-Beatmung bei der endotrachealen Intubation bezüglich der Vermeidung einer Hypoxämie gezeigt werden $(p=0,01)$. Die Aspirationsrate im Rahmen der Intubation unterschied sich nicht signifikant zwischen den beiden Gruppen $(p=0,41)$ (• Abb. 1; [24]).

Eine Beutel-Maske-Beatmung zwischen Narkoseeinleitung und Intubation bei Patienten auf Intensivstation kann die Patientensicherheit durch signifikan- 
te Reduktion einer schweren Hypoxämie ohne begleitende Erhöhung des Aspirationsrisikos verbessern. Jedoch wurden Schwangere und Patienten mit hohem Risiko für eine Aspiration durch eine mechanische Passagestörung von der Studie ausgeschlossen. Besonders bei Hochrisikopatienten sollte also eine kritische Abwägung zwischen Aspirationsund Hypoxämierisiko erfolgen.

\section{Originalpublikation}

Chu DK, Kim LH, Young PJ et al (2018) Mortality and morbidity in acutely ill adults treated with Liberal Versus Conservative Oxygen Therapy (IOTA): a systematic review and meta-analysis. Lancet 391:1693-1705

Sauerstoff ist eines der am häufigsten (und vielerorts noch immer unkritisch) verwendeten Medikamente bei (kritisch kranken) Patienten. Die 2018 veröffentliche IOTA-Metaanalyse untersuchte die Effekte einer liberalen Sauerstofftherapie, verglichen mit einer konservativen Therapie, auf die Sterblichkeit und Morbidität von akut erkrankten Patienten [25]. In die Analyse wurden 25 randomisierte, kontrollierte Studien mit insgesamt 16.037 Patienten eingeschlossen. Es wurden chirurgische und konservative Patienten mit folgenden Grunderkrankungen untersucht: Sepsis, Myokardinfarkt, Schlaganfall, Herzstillstand, Schädel-Hirn-Trauma, Beinischämie und Hohlorganperforation. Die Metaanalyse zeigte eine signifikant geringere Sterblichkeit bei Patienten, die eine konservative Sauerstofftherapie mit niedrigeren Zielwerten für die periphere Sauerstoffsättigung erhalten hatten. Die eingeschlossenen chirurgischen Patienten entwickelten unter liberaler Sauerstofftherapie zwar signifikant weniger im Krankenhaus erworbene Infektionen, jedoch ohne Einfluss auf die Sterblichkeit. Sonstige Parameter wurden durch die Sauerstofftherapie aber nicht beeinflusst. Die Autoren der Studie geben als Schwellenwert für die periphere Sauerstoffsättigung, ab dem eine Sauerstoffgabe das Behandlungsergebnis negativ beeinflusst, einen Wert von 94-96\% an.

Sauerstoff ist ein wichtiges Medikament bei der Behandlung einer Hypox- ämie. Wie bei jedem Medikament ist die richtige Dosierung jedoch entscheidend, um den Patienten durch dessen Gabe nicht zu schädigen. Es sollte die minimal mögliche Dosierung gewählt werden, um einen adäquaten Zielwert für die Sauerstoffsättigung von max. 94-96\% zu erreichen.

\section{Zusammenfassung: Beatmung und Sauerstofftherapie}

Die Studie Extracorporeal Membrane Oxygenation for Severe Acute Respiratory Distress Syndrome (EOLIA) konnte keinen Überlebensvorteil für die Therapie mithilfe der extrakorporalen Membranoxygenierung (ECMO) beim schweren ,acute respiratory distress syndrome" (ARDS) zeigen. Jedoch ergeben sich Hinweise, dass die Anwendung der ECMO das Überleben der Patienten verbessern könnte. Wenn eine lungenprotektive Beatmung nicht aufrechterhalten werden kann, sollte die Anwendung einer ECMO-Therapie in Betracht gezogen werden. Bei Patienten ohne ARDS zeigte die Anwendung kleiner Tidalvolumina gegenüber mittleren Tidalvolumina keine Vorteile. Ein nichtinvasives WeaningProtokoll erwies sich als sicher bei reduziertem Bedarf an invasiver Beatmung und Antibiotika für die Therapie respiratorischer Infektionen, ohne die Gesamtbeatmungsdauer zu verkürzen. Bei immunsupprimierten Patienten zeigte die High-flow-Sauerstofftherapie keinen Überlebensvorteil gegenüber einer Standardsauerstofftherapie. Eine BeutelMaske-Beatmung zwischen Narkoseeinleitung und Intubation bei Patienten auf Intensivstation kann die Patientensicherheit durch Prävention schwerer Hypoxämien ohne begleitende Erhöhung des Aspirationsrisikos verbessern. Eine dauerhafte, übermäßige Sauerstoffgabe bei kritisch kranken Patienten sollte vermieden werden. Zielwerte der Sauerstoffsättigung über 94-96\% sollten nicht angestrebt werden.

\section{Korrespondenzadresse}

Univ.-Prof. Dr. med. M. A. Weigand

Klinik für Anästhesiologie, Universitätsklinikum Heidelberg

Heidelberg, Deutschland

markus.weigand@med.uni-heidelberg.de

Interessenkonflikt. M. Dietrich, C.J. Reuß, C. Beynon, A. Hecker, C. Jungk, D. Michalski, C. Nusshag,

K. Schmidt, M. Bernhard, T. Brenner und M.A. Weigand geben an, dass kein Interessenkonflikt besteht.

\section{Literatur}

1. Rhodes A et al (2017) Surviving sepsis campaign: international guidelines for management of sepsis and septicshock:2016. Crit Care Med 45(3):486-552

2. Ranieri VM et al (2012) Acute respiratory distress syndrome: the Berlin definition. JAMA 307(23):2526-2533

3. Force ADT et al (2012) Acute respiratory distress syndrome: the Berlin definition. JAMA 307(23):2526-2533

4. Bellani G et al (2016) Epidemiology, patterns of care, and mortality for patients with acute respiratory distress syndrome in intensive care units in 50 countries. JAMA 315(8):788-800

5. Sud $S$ et al (2010) Prone ventilation reduces mortality in patients with acute respiratory failure and severe hypoxemia: systematic review and meta-analysis. Intensive Care Med 36(4):585-599

6. Mercat A et al (2008) Positive end-expiratory pressuresettinginadultswithacutelunginjuryand acute respiratory distress syndrome: a randomized controlled trial.JAMA 299(6):646-655

7. Fujishima S (2014) Pathophysiology and biomarkers of acute respiratory distress syndrome. JIntensive Care 2(1):32

8. Fachgesellschaften, A.d.W.M (2017) S3-Leitlinie: Invasive Beatmung und Einsatz extrakorporaler Verfahren bei akuter respiratorischer Insuffizienz. http://www.awmf.org/uploads/tx_szleitlinien/ 001-021I_S3_Invasive_Beatmung_2017-12.pdf (Erstellt:4.Dez. 2017).Zugegriffen:20.März 2018

9. Sauer CM, Yuh DD, Bonde P (2015) Extracorporeal membrane oxygenation use has increased by 433 $\%$ in adults in the United States from 2006 to 2011 Asaio J61(1):31-36

10. Ratnani I et al (2018) The role and impact of Extracorporeal membrane oxygenation in critical care. Methodist Debakey Cardiovasc J 14(2):110-119

11. Peek GJ et al (2009) Efficacy and economic assessment of conventional ventilatory support versus extracorporeal membrane oxygenation for severe adult respiratory failure (CESAR): a multicentre randomised controlled trial. Lancet 374(9698):1351-1363

12. Combes A et al (2018) Extracorporeal membrane oxygenation for severe acute respiratory distress syndrome. N Engl J Med 378(21):1965-1975

13. Bernard GR et al (1994) The American-European Consensus Conference on ARDS. Definitions, mechanisms, relevant outcomes, and clinical trial coordination. Am J Respir Crit Care Med 149(3 Pt 1):818-824

14. Goligher EC et al (2018) Extracorporeal membrane oxygenation for severe acute respiratory distress syndrome and posterior probability of mortality 
benefitin a posthoc Bayesian analysis of a randomized clinical trial. JAMA 320(21):2251-2259

15. Petrucci N, De Feo C (2013) Lung protective ventilation strategy for the acute respiratory distress syndrome. Cochrane Database Syst Rev 2:CD3844

16. Writing Group for the, P.I. et al (2018) Effect of a low vs intermediate tidal volume strategy on ventilator-free days in intensive care unit patients without ARDS: a randomized clinical trial. JAMA 320(18):1872-1880

17. Beitler JRetal (2019) Effect of titrating positive endexpiratory pressure (PEEP) with an esophageal pressure-guided strategy vs an empirical high PEEP-Fio2 strategy on death and days free from mechanical ventilation among patients with acute respiratory distress syndrome: a randomized clinical trial. JAMA 321(9):846-857

18. Talmor D et al (2008) Mechanical ventilation guided by esophageal pressure in acute lung injury. N Engl J Med 359(20):2095-2104

19. Ferguson ND et al (2013) High-frequency oscillation in early acute respiratory distress syndrome. NEngl J Med 368(9):795-805

20. Girard TD et al (2017) An official American thoracic society/American college of chest physicians clinical practice guideline: liberation from mechanical ventilation in critically ill adults. Rehabilitation protocols, ventilator liberation protocols, and cuff leak tests. Am J Respir Crit Care Med 195(1):120-133

21. Perkins GD et al (2018) Effect of Protocolized weaning with early Extubation to Noninvasive ventilation vs invasive weaning on time to liberation from mechanical ventilation among patients with respiratory failure: the breathe randomized clinical trial.JAMA320(18):1881-1888

22. Frat JP etal (2015) High-flow oxygen through nasal cannula in acute hypoxemic respiratory failure. N Engl J Med 372(23):2185-2196

23. Azoulay $E$ et al (2018) Effect of high-flow nasal oxygen vs standard oxygen on 28-day mortality in Immunocompromised patients with acute respiratory failure: the HIGH randomized clinical trial.JAMA 320(20):2099-2107

24. Casey JD et al (2019) Bag-mask ventilation during tracheal Intubation of critically ill adults. $N$ Engl J Med 380(9):811-821

25. Chu DK et al (2018) Mortality and morbidity in acutely ill adults treated with liberal versus conservative oxygen therapy (IOTA): a systematic review and meta-analysis. Lancet 391(10131):1693-1705

Veranstaltungstipp

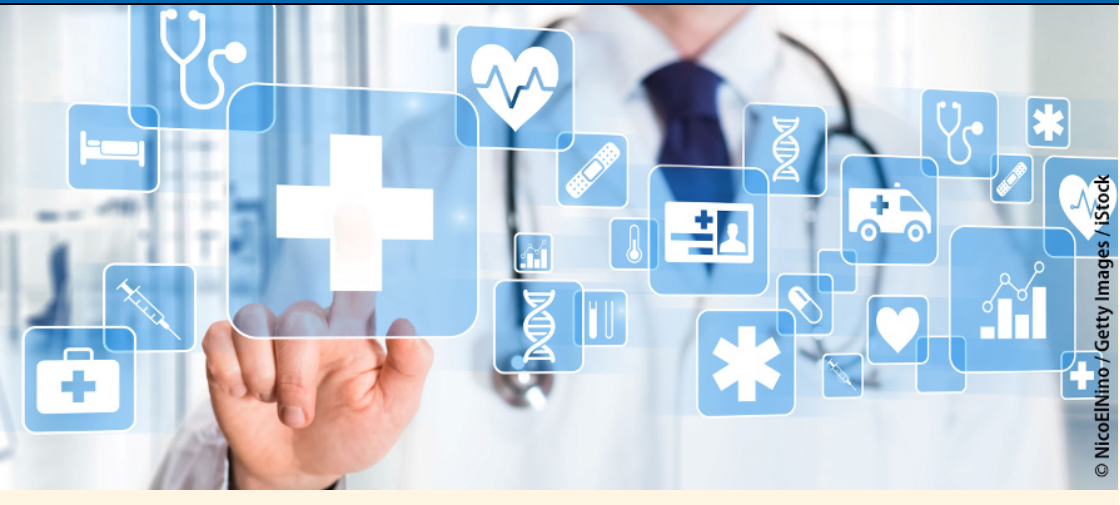

\section{Faire Bedingungen für integrierte Versorgungsformen}

\section{DGIV-Bundeskongress, 26. November 2019}

Mit den Initiativen für das „Faire-KassenwahlGesetz" wurde der Fairness-Begriff in die aktuelle gesundheitspolitische Diskussion eingebracht. Der Bundeskongress 2019 der Deutschen Gesellschaft für Integrierte Versorgung im Gesundheitswesen e.V. (DGIV) greift das auf, indem er ganz aktuell die Frage nach fairen wettbewerblichen Bedingungen im Schnittstellenbereich von ambulant und stationär stellt. Vertreter von Politik, Selbstverwaltung und Versorgungsforschung werden sich mit folgenden Themen auseinandersetzen:

- Anforderungen an einen einheitlichen Ordnungsrahmen im Schnittstellenbereich von ambulant und stationär

- Weiterentwicklung der Bedarfsplanung bei der Versorgung in den Regionen

- Vorschläge für ein modernes Vergütungssystem

- Perspektiven für die sektorenübergreifende Versorgung

- Digitalisierung des Gesundheitswesen die Roadmap der Bundesregierung

Weitere Informationen zum Programm und Anmeldeformular finden Sie unter www.dgiv.org

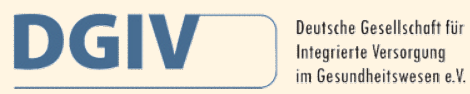

Veranstalter: DGIV e.V., Friedrichstraße 171, 10117 Berlin, Tel.: 030/44727080, Fax: 030/44729746, E-Mail: info@dgiv.org

\section{Wissenschaftliche Leitung}

Prof. Dr. Stefan G. Spitzer, Dresden

Prof. Dr. Dr. Alfred Holzgreve, Berlin

Dr. Michael Meyer, Berlin

Tagungsort: Hotel Aquino, Tagungszentrum Katholische Akademie Hannoversche Straße 5b, 10115 Berlin-Mitte

DGIV-Fachdiskussion am 25. November 2019, 18 bis 21 Uhr

Ein dritter Versorgungsbereich - Zwischenschritt zur echten integrierten Versorgung?

SpiFa, KBV, Bund/Länder-AG, Notfallversorgung - die Konzepte häufen sich, in denen ein dritter Versorgungsbereich zwischen ambulant und stationär mehr oder minder konkrete Ausgestaltung erfährt, um die Sektorengrenze zu überwinden. Die DGIV e.V. sieht in diesen unterschiedlichen Konzepten interessante Ansätze, um zu echten integrierten Versorgungslösungen kommen zu können. Wie aber könnten die vorliegenden Konzepte weitergedacht werden, zu einem Versorgungs- und Vergütungssystem ohne Sektorengrenzen zu kommen? Dieser Frage widmet sich die Fachdiskussion am Vorabend des DGIV-Bundeskongresses.

Veranstaltungsort: Hauptstadtbüro der Siemens Healthineers AG, Friedrichstr. 133, 10117 Berlin

Quelle und weitere Infos: www.dgiv.org 